\title{
Photocatalytic activity of twist-angle stacked 2D $\mathrm{TaS}_{2}$
}

Evgeniya Kovalska*, Pradip Kumar Roy, Nikolas Antonatos, Vlastimil Mazanek, Martin Vesely, Bing Wu, Zdenek Sofer*

University of Chemistry and Technology Prague, Technická 5, 16628 Prague 6, Czech Republic

ABSTRACT: The development of low-cost efficient photoelectrosensitive electrodes as an alternative to the expensive and complex rigid systems is yet in huge demand for advanced photoresponsive technology. Herein, the light-induced efficiency of electrochemically exfoliated $\mathrm{TaS}_{2}$ nanosheets for hydrogen generation catalysis and photodetectors has been demonstrated for the first time. The electrochemical exfoliation of $\mathrm{TaS}_{2}$ crystals toward a few-layer derivative has been pioneered in anhydrous tetrabutylammonium hexafluorophosphate in $\mathrm{N}, \mathrm{N}$-dimethylformamide. Comprehensive analysis of as-exfoliated $\mathrm{TaS}_{2}$ revealed the formation of nanoparticles and nanosheets with a lateral size of about several nanometers and micrometers, correspondingly. Observed mutual twisting of $2 \mathrm{H}-\mathrm{TaS}_{2}$ flakes leads to the redistribution of charge density induced by interlayer interaction of the individual nanosheets. External light irradiation on the $\mathrm{TaS}_{2}$ surface influences its conductivity making the material feasible for photoelectrocatalysis and photodetection. The $\mathrm{TaS}_{2}$-based catalyst demonstrates high HER photoelectrocatalytic activity with the onset overpotential below $575 \mathrm{mV}$ vs. RHE which can be lowered by thorough catalyst preparation. Finally, the $\mathrm{TaS}_{2}$-integrated photodetector in the acidic medium represents its broadband light sensing capability with the highest photoresponsivity $\left(0.68 \mathrm{~mA} \mathrm{~W}^{-1}\right)$ toward 420 
$\mathrm{nm}$ light illumination. This finding will pave the way to a new realization of exfoliated twist-angle stacked $\mathrm{TaS}_{2}$ for photo-induced electrochemistry and sensing.

\section{INTRODUCTION}

With increasing demands in modern society, the energy crisis and environmental problems are currently on the spotlight. Hydrogen is found to be the main sustainable source for renewable energy and is highly required for advanced energy conversion systems. Recently, photoelectrocatalytic and photoelectrochemical water-splitting methods are found as an efficient approach for the scalable generation of hydrogen. The concept of light-induced energy production through semiconductor catalysts attracted many research efforts aiming at the development of lowcost efficient bifunctional materials for hydrogen production and environment-sensing response. The performance of these materials can be tuned while illuminating the light of various wavelengths. The occurred photo-induced electron transfer in the targeted nanosheets-based sensitive material, will generate photoelectrochemical water-splitting e.g. faster hydrogen evolution reaction (HER) or broadband light-sensitive detector. These will pave new ways for emerging materials as a cheaper and more productive alternative to most common fossil fuelsbased methods of hydrogen production as well as traditional photodetectors.

Two-dimensional (2D) transition-metal dichalcogenides (TMDs) have been demonstrated as promising catalysts for water splitting, hydrogen reduction and water oxidation. ${ }^{1,2}$ The unique chemical and physical properties of 2D TMDs are demonstrated by their small size enriched by the number of edge active sites, band-edge position, quantum confinement effect and thus, exhibit photo-induced catalytic efficiency. ${ }^{3}$ TMDs are considered as the most promising cost-effective catalysts and their properties are determined by the TMDs' polymorph type namely hexagonal $2 \mathrm{H}$ 
or trigonal $1 \mathrm{~T}$. It has been reported that stable $2 \mathrm{H}-\mathrm{MoS}_{2}$ shows semiconducting properties meanwhile metastable $1 \mathrm{~T}-\mathrm{MoS}_{2}{ }^{4}$ or $1 \mathrm{~T}-\mathrm{WS}_{2}{ }^{5}$ forms demonstrate metallic properties. The metallic phase of a material is capable of a more efficient performance than the semiconducting $2 \mathrm{H}$ counterpart owing to its enhanced electrical conductivity. The metallic character is associated with undercoordinated chalcogen atoms at the 1010 edge of 2D TMD providing for instance more efficient adsorption of hydrogen which is a crucial step for electrochemical water splitting ${ }^{6}$. The $\mathrm{MoS}_{2}$ and $\mathrm{WS}_{2}$ exhibit superior catalytic activity and are used as alternatives to Pt-based catalysts ${ }^{7-}$ 9. The $\mathrm{WSe}_{2}$ is demonstrated as an excellent output stability photodetector due to its high absorption coefficient in the visible and near-infrared regions ${ }^{10,11}$. Other known $2 \mathrm{D}$ materials such as $\mathrm{TiSe}_{2},{ }^{12-14} \mathrm{NiSe}_{2}{ }^{15,16} \mathrm{TaSe}_{2}{ }^{17}$ and $\mathrm{TaS}_{2}{ }^{18-20}$ are interesting due to their superconductivity, charge-density-wave and metal-insulator transitions.

Among other 2D TMD materials, tantalum disulfide $\left(\mathrm{TaS}_{2}\right)$ has been subject to numerous studies due to the variety of the material's unique structural and electronic phases. ${ }^{21}$ Its initial metallicity and thus, electrical conductivity can lead to potential applications such as a lightresponsive active electrode in the HER and photosensing. Albeit, the study of $\mathrm{TaS}_{2}$ is mainly focused on the superconductivity, ${ }^{22-24}$ field emission ${ }^{25,}{ }^{26}$, meanwhile, photo-induced features of the material for HER and photosensing have not been explored yet. Aiming to enhance the photoresponse performance of the $\mathrm{TaS}_{2}$, the quality and dimensions of the material must be considered. For instance, bulk $\mathrm{TaS}_{2}$ possesses a lack of exposed active sites that significantly affect the conversion efficiency of the material and narrows its application. Therefore, a thorough cleavage of the van der Waals structure by selecting the most appropriate method is essential to increase the conversion efficiency of $\mathrm{TaS}_{2}$. 
Several methods have been demonstrated to produce layered $\mathrm{TaS}_{2}$ such as chemical vapor deposition (CVD), ${ }^{27}$ mechanical cleavages, ${ }^{28}$ intercalation, ${ }^{29}$ ultrasonication ${ }^{30-32}$, or liquid-phase exfoliation. ${ }^{22}$ However, the CVD method remains to be improved before it can be used to produce large-domain homogeneous $\mathrm{TaS}_{2}$ films. The mechanical cleavage is not scalable and undergoes a lack of control over product thickness and size. Alternatively, electrochemical exfoliation (one of the solution-based techniques) is the most convenient, simple and controlled method that can be employed in ambient temperature for large-scale production of $2 \mathrm{D} \mathrm{TaS}_{2}$. Recently the electrochemical approach has been successfully applied for the exfoliation of a few-layer phosphorene ${ }^{33}$ and arsenene $e^{34}$ and has been pioneered for single-step production of platinoiddecorated phosphorene. ${ }^{35}$

Therefore, in this study, the photoresponse of electrochemically exfoliated $\mathrm{TaS}_{2}$ nanosheets and preliminarily characterized by micro- and spectroscopic techniques (Supporting Information: N1) was investigated in an effort to develop a low-cost efficient photosensitive electrode that can replace the noble metal-based catalysts ${ }^{36}$ (as photoelectrocatalyst - PEC) and CMOS-based photodetectors ${ }^{37}$ (as photodetector - PD). Pioneered low-potential electrochemical exfoliation of $\mathrm{TaS}_{2}$ crystals was carried out in the non-aqueous electrolyte medium of tetrabutylammonium hexafluorophosphate $\left(\mathrm{TBAPF}_{6}\right)$ in $\mathrm{N}, \mathrm{N}$-dimethylformamide (DMF). The as-exfoliated 2D $\mathrm{TaS}_{2}$ nanostructures consist of the few-layer nanosheets and nanoparticles. Detailed characterization of bulk and exfoliated $\mathrm{TaS}_{2}$ was performed through various microscopic and spectroscopic techniques. The photo-induced high HER electrocatalytic activity of the as-exfoliated $\mathrm{TaS}_{2}$ nanostructures with low overpotential ( $>575 \mathrm{mV}$ and $320 \mathrm{mV}$ ) was reported. A room-temperature $\mathrm{TaS}_{2}$-based broadband photodetector in the acidic medium of $0.5 \mathrm{M} \mathrm{H}_{2} \mathrm{SO}_{4}$ has been presented. The higher electrical conductivity of $2 \mathrm{D} \mathrm{TaS}_{2}$ compared to its bulk form can improve the efficiency 
of charge distribution in the catalytic/sensing systems and, thus, broaden the material's application area.

\section{RESULTS}

\section{(I) Synthesis and characterization of the materials}

Electrochemical exfoliation of bulk $\mathrm{TaS}_{2}$ crystal (see scanning electron microscopy (SEM) images in Figures S1 a-c) was performed in the anhydrous electrolyte of $0.01 \mathrm{M} \mathrm{TBAPF}_{6}$ in $\mathrm{DMF}$ Following three main steps of the procedure (Scheme 1) namely wetting of the crystal, accumulation (or activation) of the tetrabutylammonium cations $\left(\mathrm{TBA}^{+}\right)$and their intercalation between $\mathrm{TaS}_{2}$ layers were carried out at $-1,-2.5$ and $-3.8 \mathrm{~V}$ (vs. $0.1 \mathrm{M} \mathrm{Ag} / \mathrm{AgNO}_{3}$ ), respectively. The first two steps were completed in 2 min each, followed by 4 hours of $\mathrm{TBA}^{+}$-assisted intercalation-delamination process at a potential of $-3.8 \mathrm{~V}$. It has to be noted that the duration of the process is defined by the initial size of the $\mathrm{TaS}_{2}$ crystal and can be terminated when a sufficient amount of the exfoliated material is produced.

After the exfoliation of the $\mathrm{TaS}_{2}$ crystal, flakes of various sizes and thicknesses were observed based on AFM analysis. These include $\mathrm{TaS}_{2}$ nanosheets of lateral size up to $6 \mu \mathrm{m}$ with a thickness of $30 \mathrm{~nm}$ as well as $2.3 \mu \mathrm{m}$ nanosheets with $7 \mathrm{~nm}$ thickness. (Figure $1 \mathrm{a}, \mathrm{b}$ respectively). In addition, recognized $\mathrm{TaS}_{2}$ nanoparticles demonstrated a lateral size of $100-200 \mathrm{~nm}$ and thickness in the range between $0.5-3 \mathrm{~nm}$. (Figure $1 \mathrm{c}$ ). The successful exfoliation of the $\mathrm{TaS}_{2}$ crystal into nanosheets and nanoparticles as well as their size distribution were confirmed by the statistical analysis (Figure 2) obtained from AFM images. The results are also in good agreement with STEM images (Figures S1d-f) as well as TEM images coupled with EDX (Figure S2) where 
the dispersion of two-types of exfoliated nanostructures with the lateral size of about tens and hundreds of nanometers as well as several micrometers are depicted.

Scheme 1. Electrochemical exfoliation of $\mathrm{TaS}_{2}$ crystal*

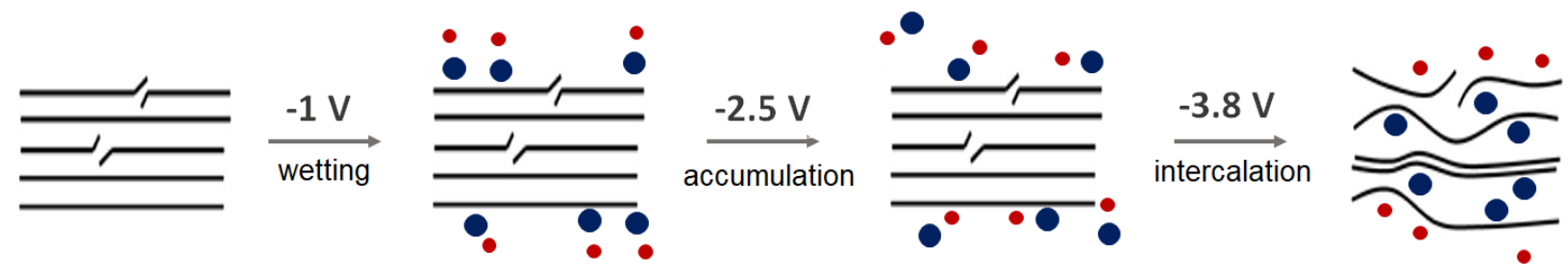

*Blue $\bullet-$ cations $\left(\mathrm{TBA}^{+}\right)$; red $\bullet-$ anions $\left(\mathrm{PF}^{-}\right)$.

TEM and SAED microscopies demonstrate good quality of exfoliated $\mathrm{TaS}_{2}$ nanosheets (Figure 3 a-d) of hexagonal structure (schematic image, Figure 3 e, f). High-resolution TEM images in Figure $\mathrm{S} 3$ also show the formation of round-shaped $\mathrm{TaS}_{2}$ nanoparticles that are localized on the surface of the larger flakes. This is consistent with the statistical analysis of particle size distribution (Figure 2) that confirms the production of two types of $\mathrm{TaS}_{2}$ nanostructures. Based on the fast Fourier transform (FFT) analysis of TEM images (Figure S4), the crystal symmetry of $\mathrm{TaS}_{2}$ is demonstrated as the fringes oriented in a three-fold rotation axis. The d-spacing of the symmetry of the $\mathrm{TaS}_{2}$ crystal of about $2.9 \AA$ and $2.93 \AA$ defines its (100) planes' orientations. The electron diffraction patterns of a flat area of the $\mathrm{TaS}_{2}$ nanosheet show this growing direction. Different magnification TEM images of the $\mathrm{TaS}_{2}$ exhibit in-plane atoms ordering with the lattice spacing $0.305 \mathrm{~nm}$. The morphology of the exfoliated material and thus sharply defined layered appearance are caused by the initial structure of the $\mathrm{TaS}_{2}$ crystal. 

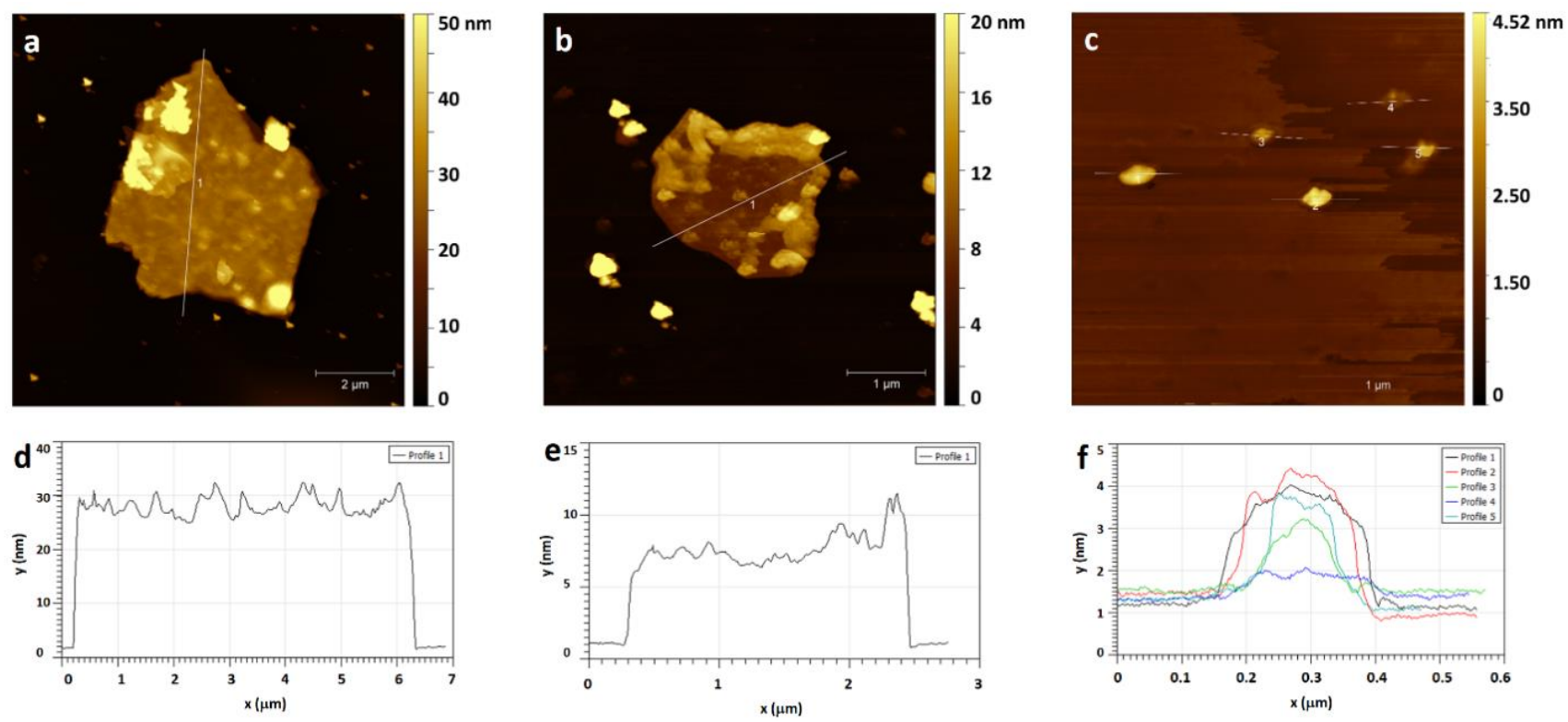

Figure 1. AFM images of the as-exfoliated $\mathrm{TaS}_{2}$ nanosheets $(\mathrm{a}, \mathrm{b})$, nanoparticles (c) and their corresponding height profiles (d-f).

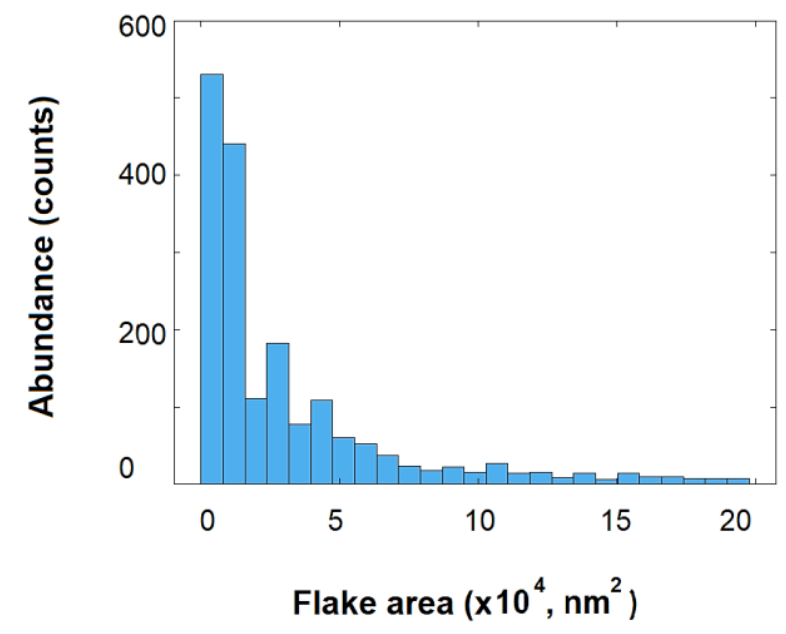

Figure 2. The statistical analysis of the particle size distribution of exfoliated $\mathrm{TaS}_{2}$ in $\mathrm{DMF}$. The evaluation was conducted based on the AFM results. 

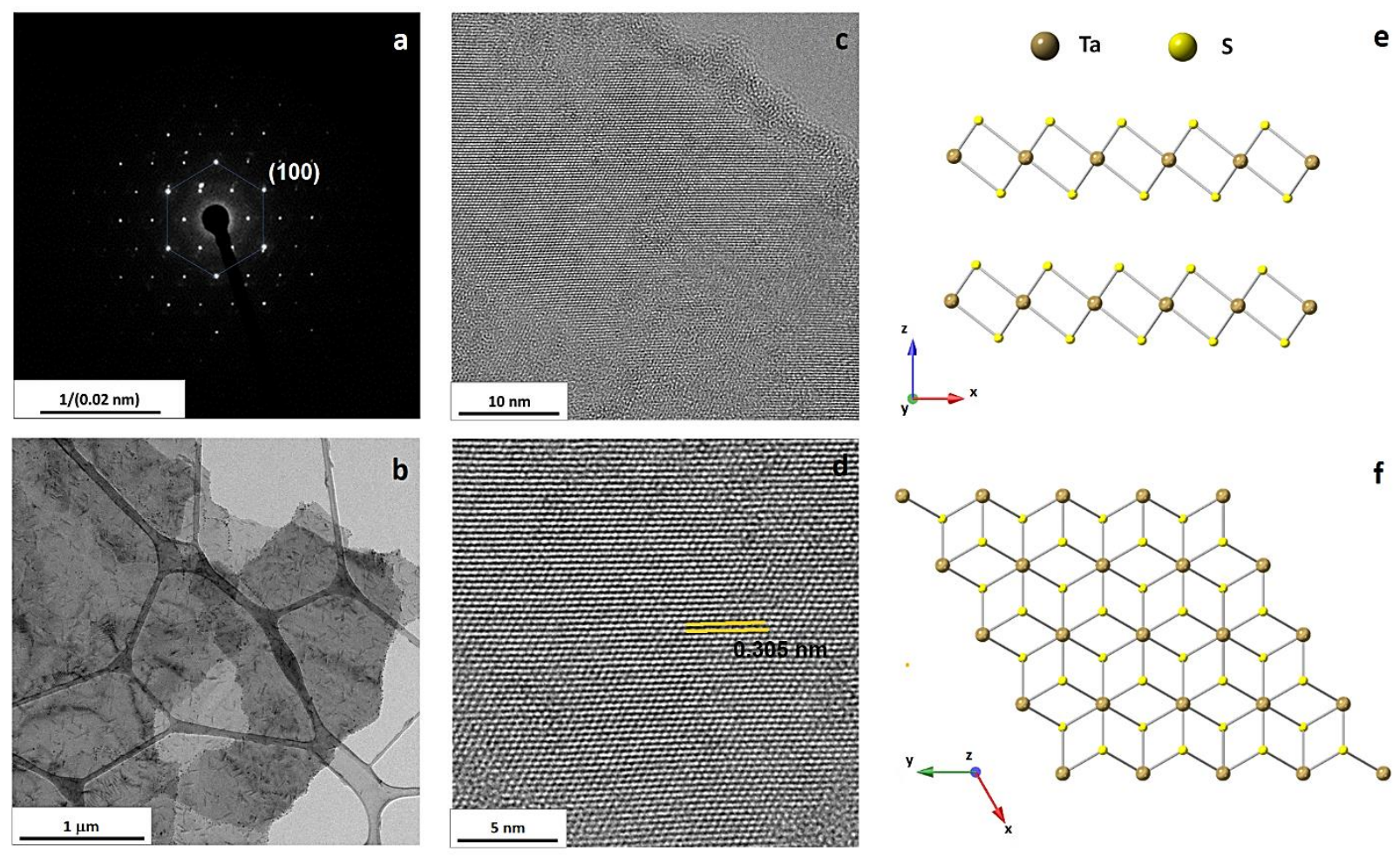

Figure 3. Morphological and structural analysis of electrochemically exfoliated $\mathrm{TaS}_{2}$. (a) SAED pattern; (b) low-resolution TEM image; (c, d) high-resolution TEM images; schematic (e) side and (f) top views of the $\mathrm{TaS}_{2}$ structure.

The SAED patterns of the large area $\mathrm{TaS}_{2}$ flakes (Figure S5) verified several configurations with their different twist angles of $7^{\circ}, 8^{\circ}, 9^{\circ}, 10^{\circ}$ (Figure 4). The trigonal arrangement of Ta atoms in Figure $3 \mathrm{e}, \mathrm{f}$ corresponds to the standard stacking of S-Ta-S in the unit cell. This direct stacking of each layer via Ta atoms was experimentally and theoretically proven in a few publications. ${ }^{38-40}$ The increase of both formation energy and bandgap has been theoretically explained due to the interlayer interaction of twisted $\mathrm{TaS}_{2}$ flakes and the redistribution of charge density induced by the in-plane distortion. ${ }^{39}$ This feature can also lead to the increase of the electronic specific-heat coefficient of the randomly twisted $\mathrm{TaS}_{2}$ nanosheets ${ }^{22}$ establishing the material as a promising candidate for photo-induced applications. ${ }^{41}$ 

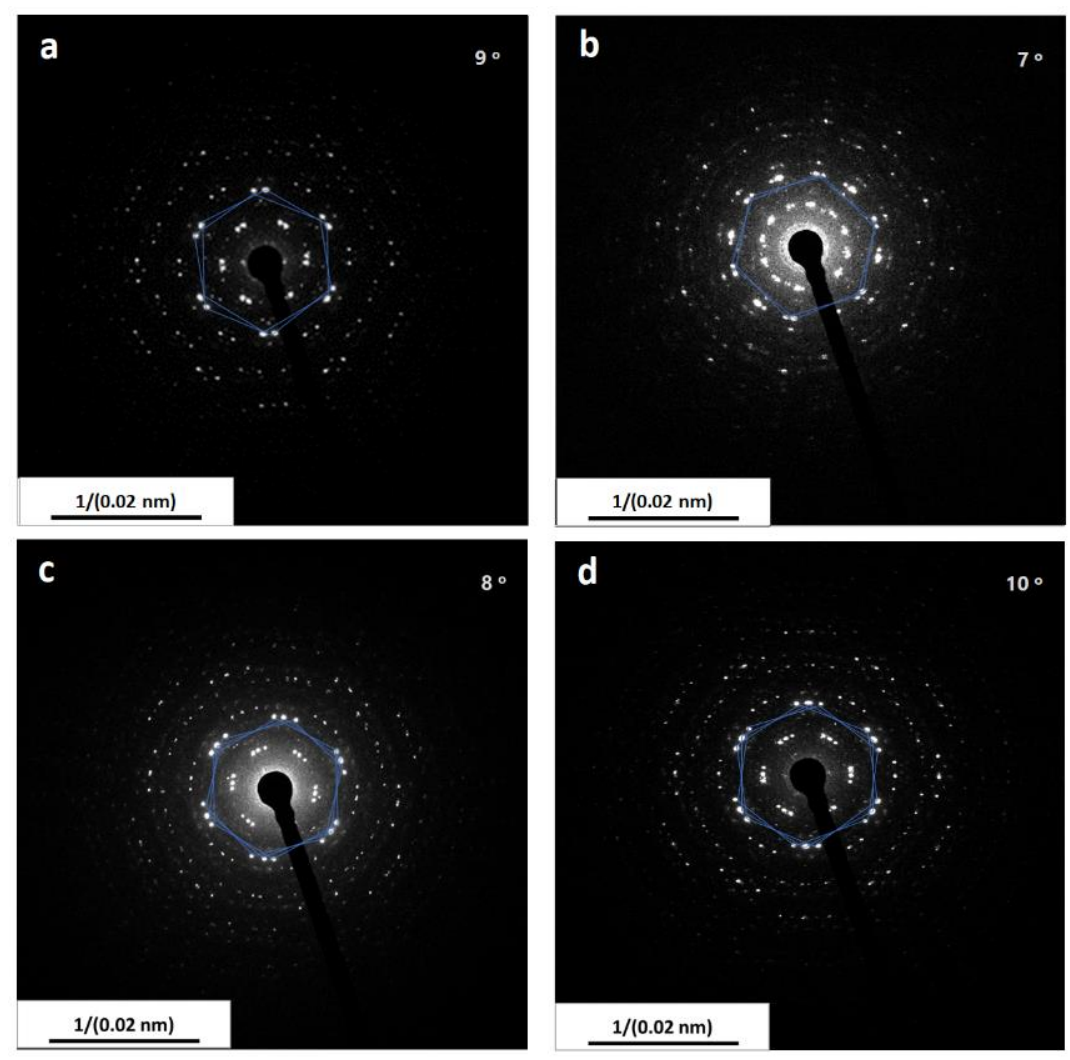

Figure 4. The SAED patterns are taken from a large area that refers to the various twist of the $(\mathrm{a}, \mathrm{b})$ bilayer and $(\mathrm{c}, \mathrm{d})$ three-layer $\mathrm{TaS}_{2}$.

The analysis of the crystal structure and optical characteristics of the bulk and exfoliated $\mathrm{TaS}_{2}$ is demonstrated in Figure 5. X-ray diffraction (XRD) was used to verify the successful synthesis of hexagonal $2 \mathrm{H}-\mathrm{TaS}_{2}$ (Supporting Information: N2, 3). The XRD pattern in Figure 5 a confirmed the $P-3 m 1$ hexagonal structure of $\mathrm{TaS}_{2}$ (PDF: 04-003-4190). No other phases were observed indicating the purity of the starting material. The sharp peaks in the pattern ascertaining the high crystallinity of the sample. Similar peaks were observed in the exfoliated material's XRD pattern albeit broadened and with lower intensity caused by the disruption of initial crystallinity due to the exfoliation procedure. The broadening of the XRD peaks is an indication of a decrease in crystal size and lattice strain increase. Figure $5 \mathrm{~b}$ shows the Raman spectra of bulk and asexfoliated $\mathrm{TaS}_{2}$ samples. Three main Raman features depicted at 239, 301 and $373 \mathrm{~cm}^{-1}$ from the 
bulk crystal were assigned to the $\mathrm{E}_{2 \mathrm{~g}}, \mathrm{E}_{1 \mathrm{~g}}$ and $\mathrm{A}_{1 \mathrm{~g}}$ modes respectively. ${ }^{29,42}$ More prominent peaks of the same modes and their $4 \mathrm{~cm}^{-1}$ redshifts were observed for the exfoliated nanosheets. The shift is attributed to the decrease of the interlayer van der Waals forces and due to the material's thickness reduction. UV-VIS absorption spectrum of the as-exfoliated $\mathrm{TaS}_{2}$ nanostructures recorded at room temperature is demonstrated in Figure $5 \mathrm{c}$. The $\mathrm{TaS}_{2}$ represents low in-plain reflectivity at high energies (from $400-720 \mathrm{~nm}$ ) and vice versa at low energies accordingly. ${ }^{43}$

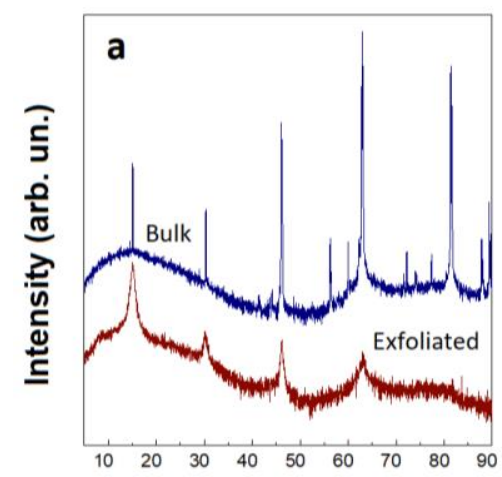

$2 \Theta$

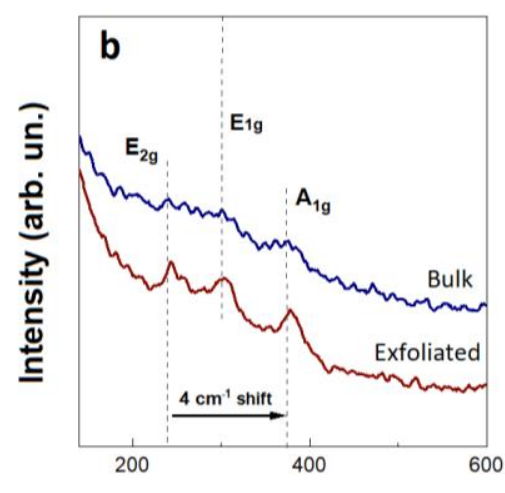

Raman shift $\left(\mathrm{cm}^{-1}\right)$

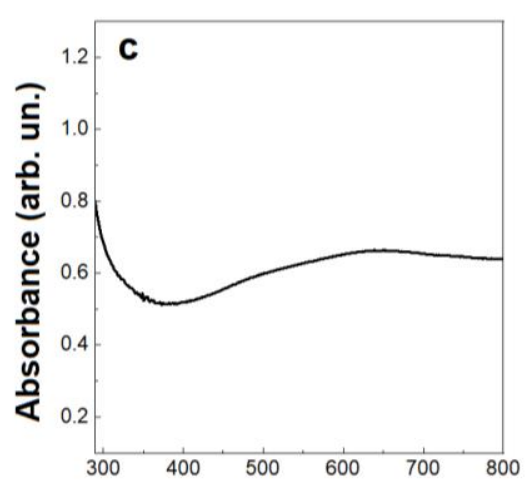

Wavelength $(\mathrm{nm})$

Figure 5. Structural and optical characteristics of the $\mathrm{TaS}_{2}$ : (a) XRD diffractogram of bulk $\mathrm{TaS}_{2}$ and electrochemically exfoliated nanosheets; (b) Raman spectra of bulk and electrochemically exfoliated $\mathrm{TaS}_{2}$; (c) UV-VIS absorbance spectra of the exfoliated $\mathrm{TaS}_{2}$ in the DMF.

To confirm chemical composition and binding states, we have measured the survey (Figure S6) and high-resolution X-ray photoelectron spectra (HR-XPS) of Ta 4f and S 2p (Figure 6). Survey spectra confirmed composition which was close to $\mathrm{TaS}_{2}$ for bulk and exfoliated samples as well. Since exfoliated samples were measured on a gold substrate, Au peaks can also be seen in its survey spectrum. HR-XPS Ta $4 \mathrm{f}$ spectra were deconvoluted with two binding states $\mathrm{Ta}^{\mathrm{IV}}\left(\mathrm{TaS}_{2}\right)$ and $\mathrm{Ta}^{\mathrm{V}}\left(\mathrm{Ta}_{2} \mathrm{O}_{5}\right)$ with binding energies 22.9 and $26.1 \mathrm{eV}$, respectively. ${ }^{44}$ Although $\mathrm{O} 2 \mathrm{~s}$ at $24.0 \mathrm{eV}$ overlaps with the Ta 4f, it was not used for deconvolution because its amount was 
less than $1 \%$ due to a low relative sensitivity factor of 0.14 compared to 8.62 for Ta 4 f. Each binding state in $\mathrm{Ta} 4 \mathrm{f}$ has spin-orbit components separated by $1.92 \mathrm{eV}$. Binding state ratios are summarized in Table S1. Bulk crystal was covered by $20 \%$ of $\mathrm{Ta}_{2} \mathrm{O}_{5}$ and after exfoliation, this amount increased to $30 \%$. It has to be noted that $\mathrm{TaS}_{2}$ is susceptible to oxidation and this ability is even higher after the exfoliation. ${ }^{44}$ Thus, operating the material in an oxygen-free environment and storing it in the dry solvents will increase the stability of as-exfoliated $\mathrm{TaS}_{2}$.
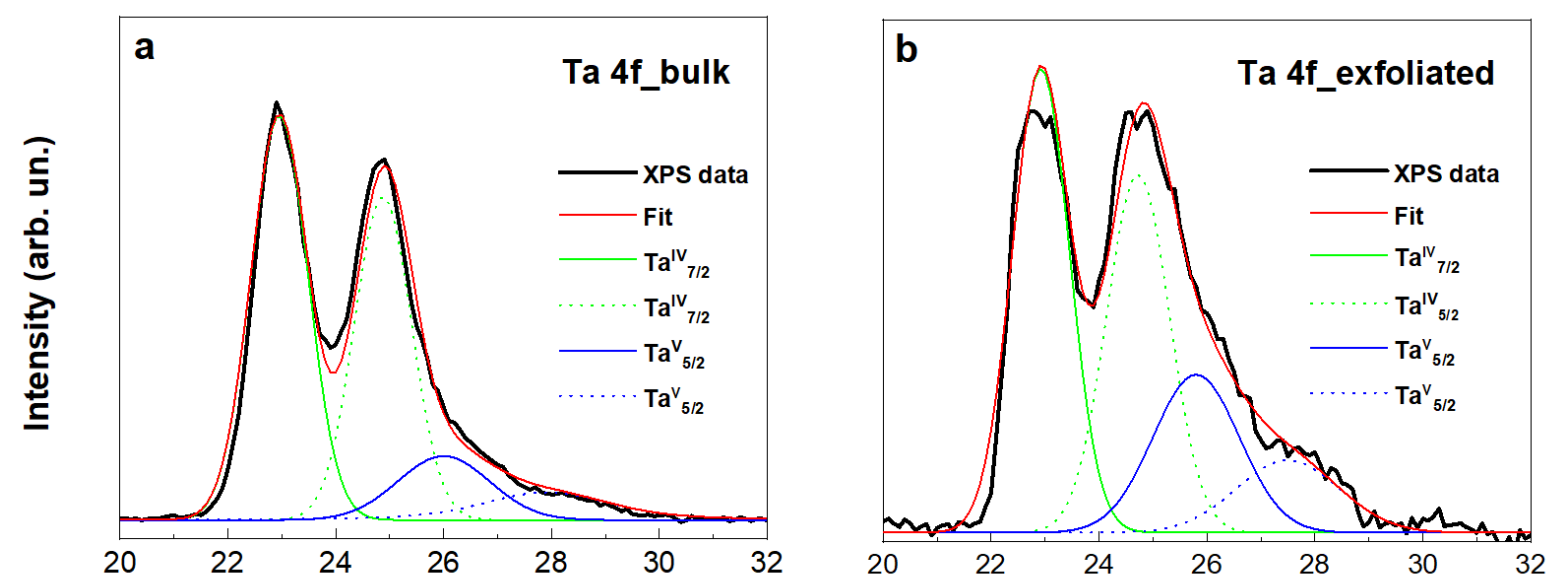

Binding energy (eV)

Figure 6. High-resolution XPS spectra of the (a) bulk and (b) electrochemically exfoliated $\mathrm{TaS}_{2}$ nanostructures.

\section{(II) Photoelectrocatalytic performance of as-exfoliated $\mathrm{TaS}_{2}$ for HER}

Light-driven electrocatalysis using semiconducting materials (e.g. $\left.\mathrm{TaS}_{2}\right)$ or their heterostructures is one of the most promising sustainable approaches for the generation of hydrogen by water splitting. ${ }^{45-47}$ Even though a thermal treatment or catalyst support texturization of the catalytic films is used to increase the number of active centers of the $\mathrm{TaS}_{2}$ flakes, these methods are time-consuming and require a high-temperature and inert gas atmosphere. ${ }^{48}$ The light- 
induced electrochemistry (photoelectrochemistry) can be a simple approach to enhance the HER activity of the $\mathrm{TaS}_{2}$. To the best of our knowledge, the photoelectrocatalytic activity of $\mathrm{TaS}_{2}$ has not been explored to date.

Herein, the photoelectrocatalytic performance of electrochemically exfoliated $\mathrm{TaS}_{2}$ for HER was explored for the first time (see Supporting Information: N4). The three-electrode linear sweep voltammetry (LSV) was performed in the $0.5 \mathrm{M} \mathrm{H}_{2} \mathrm{SO}_{4}$ employing a few-layer $\mathrm{TaS}_{2}$ deposited on a glassy carbon as a working electrode. The measurements have been repeated in the light mode for four different wavelengths $(420,532,630$ and $720 \mathrm{~nm})$. The results were compared with the dark mode for as-exfoliated $\mathrm{TaS}_{2}$ nanosheets of mixed composition. The mixed composition consists of nanosheets of various sizes and nanoparticles as was confirmed by the STEM, AFM and statistical analysis. Few-layer $\mathrm{TaS}_{2}$ samples of a smaller lateral size $(\sim 30-40 \mathrm{~nm}$, Figure S7) were obtained as follows: first, the $\mathrm{TaS}_{2}$ dispersion was centrifuged at $3500 \mathrm{rpm}$ for $10 \mathrm{~min}$; second, collected supernatant was centrifuged at $10000 \mathrm{rpm}$ for $10 \mathrm{~min}$ and the resulting precipitate was used for the test.

As shown in Figure 7 a, $b$ the HER for initial as-exfoliated $\mathrm{TaS}_{2}$ starts at a potential of $575 \mathrm{mV}$ (vs. RHE) under dark mode at a current density of $10 \mathrm{~mA} \mathrm{~cm}{ }^{-2}$. The overpotential under the illumination light of different wavelengths is shifting towards lower potentials, more specifically: 537, 549, 531 and $454 \mathrm{mV}$ when blue, green, red and far-red light-emitting diodes (LEDs) are implemented, respectively. This behavior is attributed to the carrier increment that is generated in the $\mathrm{TaS}_{2}$ nanosheets with light irradiation enhanced by the intrinsic defect (wrinkles) and mutual twisting of the individual layers. The current density of the $\mathrm{TaS}_{2}$ catalyst under dark is decreased after 100 cycles (Figure S8), which demonstrates a slow degradation of the asinvestigated catalyst. It has to be noted that the onset overpotential for the $\mathrm{TaS}_{2}$ catalyst based on 
flakes of smaller size was significantly reduced to approximately $320 \mathrm{mV}$ (Figure $7 \mathrm{c}$ ). Considering the influence of the light on the as-exfoliated samples, the $\sim 100 \mathrm{mV}$ decrement of the overpotential is expected that will make a few-layer $\mathrm{TaS}_{2}$ a superior HER photoelectrocatalyst. This study on bare $\mathrm{TaS}_{2}$ catalysts can compete with recently reported results ${ }^{49}$ where the performance of $\mathrm{TaS}_{2}$ was enhanced by the incorporation of conductive films ${ }^{50}$ or thermal treatment in the $\mathrm{H}_{2}$-environment. ${ }^{48}$ Furthermore, thoroughly prepared catalysts under light illumination will outperform the HER activity of commercial $\mathrm{Pt} / \mathrm{C}$ electrode $(\sim 40 \mathrm{mV})$ providing a cheaper alternative.
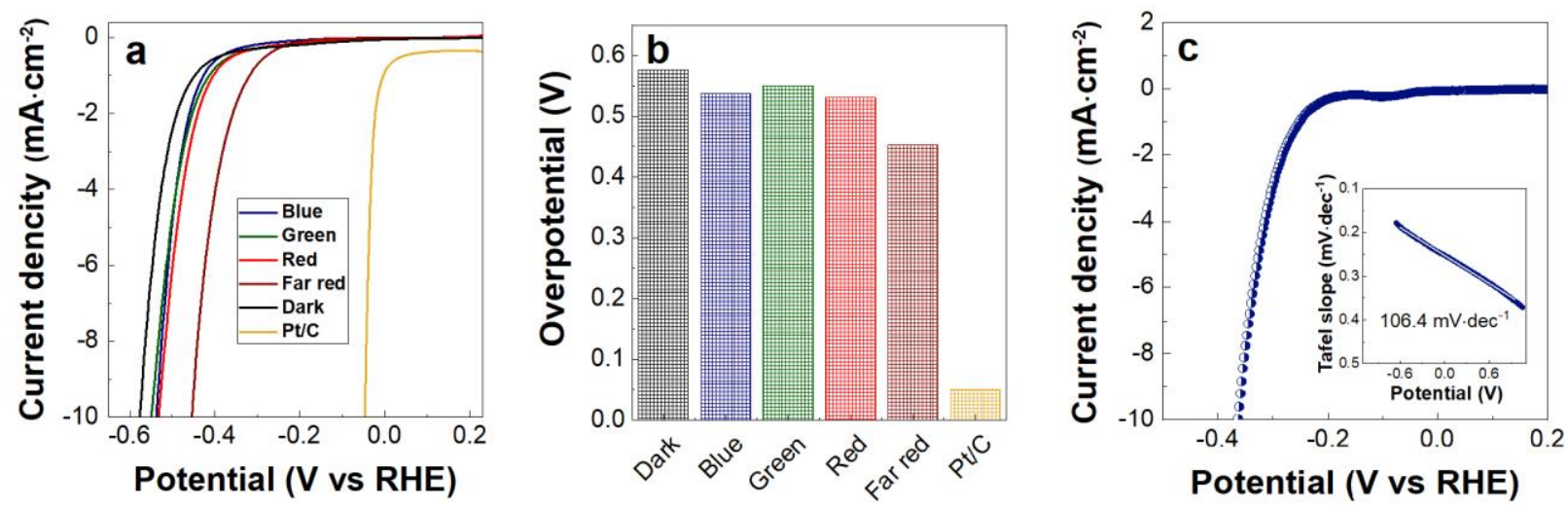

Figure 7. HER analysis in the medium of $0.5 \mathrm{M} \mathrm{H}_{2} \mathrm{SO}_{4}$ at the scan rate of $1 \mathrm{mV} \cdot \mathrm{s}^{-1}$ in dark mode and during the blue, green, red and far-red irradiation. Polarization curves (a) and corresponding overpotentials (b) of exfoliated $\mathrm{TaS}_{2}$ at a $-10 \mathrm{~mA} \cdot \mathrm{cm}^{-2}$ current density; the measurements were conducted vs. RHE and compared with commercial Pt/C electrocatalyst. Polarization curve and inserted corresponding Tafel plot (c) of few-layer $\mathrm{TaS}_{2}$ small flakes obtained after centrifugation; overpotential is $360 \mathrm{mV}$. 


\section{(III) TaS2-integrated photodetector}

Solution-based PEDs offer unique advantages over standard photodetectors in the form of a simple and easy device manufacturing process. Recently 2D nanomaterials have demonstrated their feasibility for PDs ranging from visible to THz however this is not inherent to one material. ${ }^{43}$, 51, 52 Therefore, a broadband photodetection capability of materials is highly demanded due to its wide application possibility. The intrinsic parameters of the material's light sensitivity and response can be tuned by changing the applied voltage, analyte concentration and power of the illuminated light. Herein, the photoelectrochemical response of $\mathrm{TaS}_{2}$ in $0.5 \mathrm{M} \mathrm{H}_{2} \mathrm{SO}_{4}$ acidic medium was measured in a three-electrode system at ambient temperature. The $\mathrm{TaS}_{2}$-integrated PEC was tested under four illumination wavelengths $(420,520,630$ and $720 \mathrm{~nm})$ with a scanning speed of $10 \mathrm{mV} \cdot \mathrm{s}^{-1}$. The procedure of photoelectrochemical measurements is described in Supporting Information: N5.

The results illustrated a steady response toward different illumination wavelengths as depicted in Figure 8. As shown in Figure 8 a, the power-dependent photocurrent measurement was achieved by using chronoamperometry in $0.5 \mathrm{M} \mathrm{H}_{2} \mathrm{SO}_{4}$ at $0.5 \mathrm{~V}$ against SCE under the illumination of $420 \mathrm{~nm}$ blue LED light source. The response of the $\mathrm{TaS}_{2}$-based electrode toward blue illumination was the highest in comparison with other LEDs. The response toward green illumination (Figure $8 \mathrm{~b}$ ) was decreased and slightly increased after applying red (Figure $8 \mathrm{c}$ ) and far-red (Figure $8 \mathrm{~d}$ ) light sources. The most prominent response of the electrochemically exfoliated few-layer $\mathrm{TaS}_{2}$ is demonstrated under blue illumination and well consistent with the light absorbance of the material (Figure $5 \mathrm{~d}$ ). The near-linear increment in current density with the increase of the illumination power from 100 to $1200 \mathrm{~mW}$ was illustrated (Figure S9) for all lights. 
As the intensity increases, the current response jumps to a higher value corresponding to a higher conductance, indicating that the separation of electron-hall pairs is triggered.
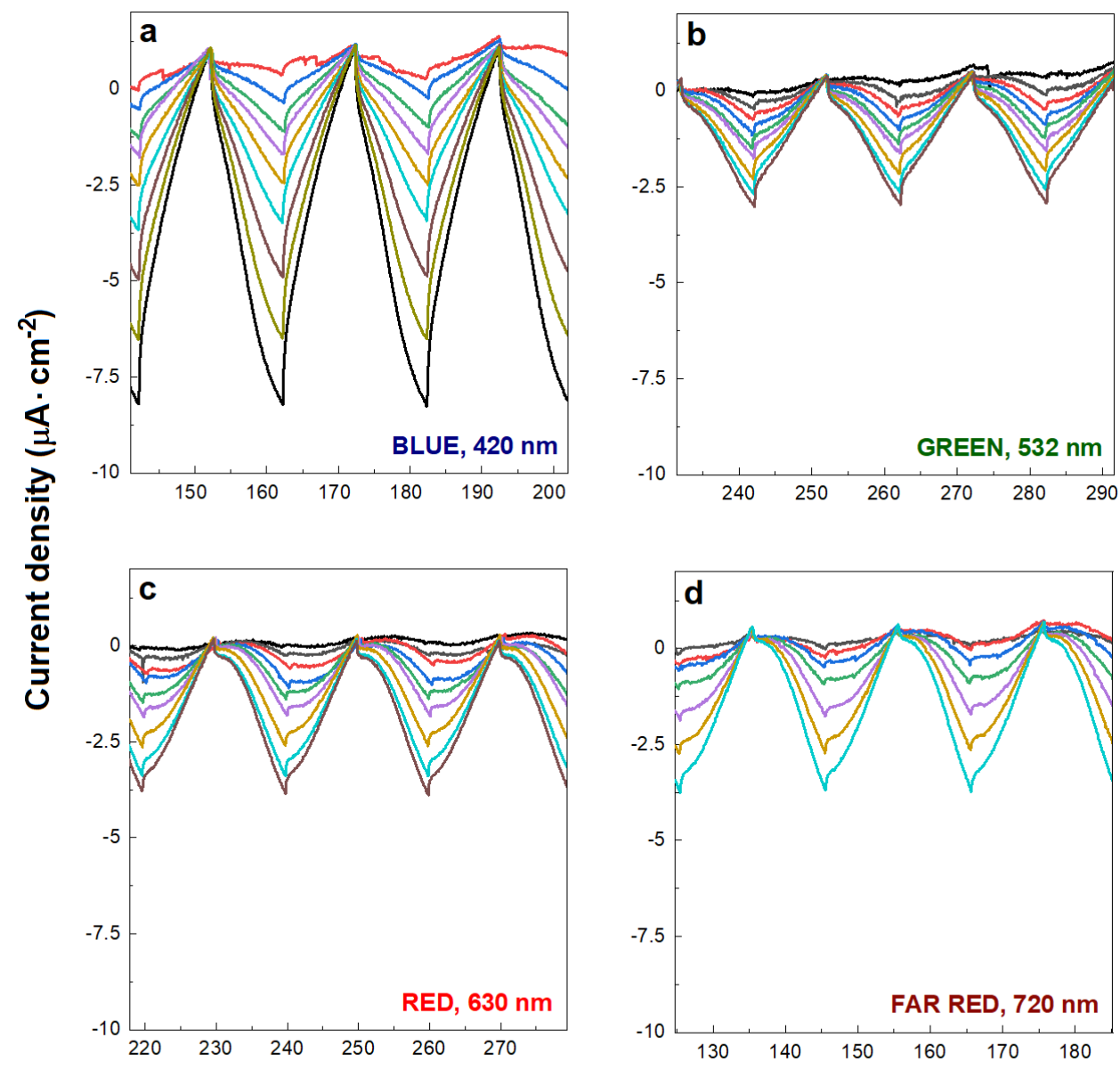

Time (sec)

Figure 8. Power dependence of photocurrent density under the illumination of (a) blue (420 nm), (b) green $(532 \mathrm{~nm})$, (c) red $(630 \mathrm{~nm})$ and (d) far red $(720 \mathrm{~nm})$ LED sources in $0.5 \mathrm{M} \mathrm{H}_{2} \mathrm{SO}_{4}$ solution at $0.5 \mathrm{~V}$ vs. SCE. The power increased from 100 to $1200 \mathrm{~mW}$ (an arrow demonstrates the direction of power increment). 
The photodetection potential of the material has been assessed in more detail by calculating a few essential parameters. At first, the photoresponsivity $\left(R_{p h}\right)$ of the $\mathrm{TaS}_{2}$-integrated PD has been calculated using the following equation: $R_{p h}=\Delta I / P$, where $\Delta I$ represents the difference in photocurrent density between the dark and photocurrent, and $P$ is the irradiation power intensity per unit area. The calculated photoresponse demonstrated the highest responsivity of about $0.62 \mathrm{~mA} \cdot \mathrm{W}^{-1}$ under the illumination of $420 \mathrm{~nm}$ blue LED source at $0.5 \mathrm{~V}$ applied potential (vs. SCE). Figure 9a highlighted the photoresponsivity of the devices concerning the power density for different illumination wavelengths. Another important parameter for the evolution of photodetection capability is specific detectivity; it has been determined accordingly $y^{53}$

$$
D^{*}=\frac{(A B)^{1 / 2}}{N E P}
$$

Eq. 1.

In the above equation $A, B$, and $N E P$ stand for the active area, measured bandwidth, and noise equivalent power, respectively. $N E P$ is defined as the input signal power resulting in a signalto-noise ratio $(S / R)$ of 1 in a $1 \mathrm{~Hz}$ output bandwidth. Furthermore, in the equation form, $N E P$ can be presented as ${ }^{53,54}$

$$
N E P=\frac{I_{N}}{R_{p h}}
$$

Eq. 2.

Here, $R_{p h}$ and $I_{N}$ represent the photoresponsivity of the PD and the noise current, respectively. In addition, the noise, which is associated with the dark current $\left(I_{D}\right)$ of the PD current, can be ascribed as $I_{N}{ }^{2}=2 e I_{D} B$, where $e$ represents the electronic charge. ${ }^{43}$ The calculated specific detectivity of our PD devices of about $1.28 \times 10^{9} \mathrm{~cm} \cdot \mathrm{Hz}^{1 / 2} \mathrm{~W}^{-1}$ (Figure $9 \mathrm{~b}$ ). 

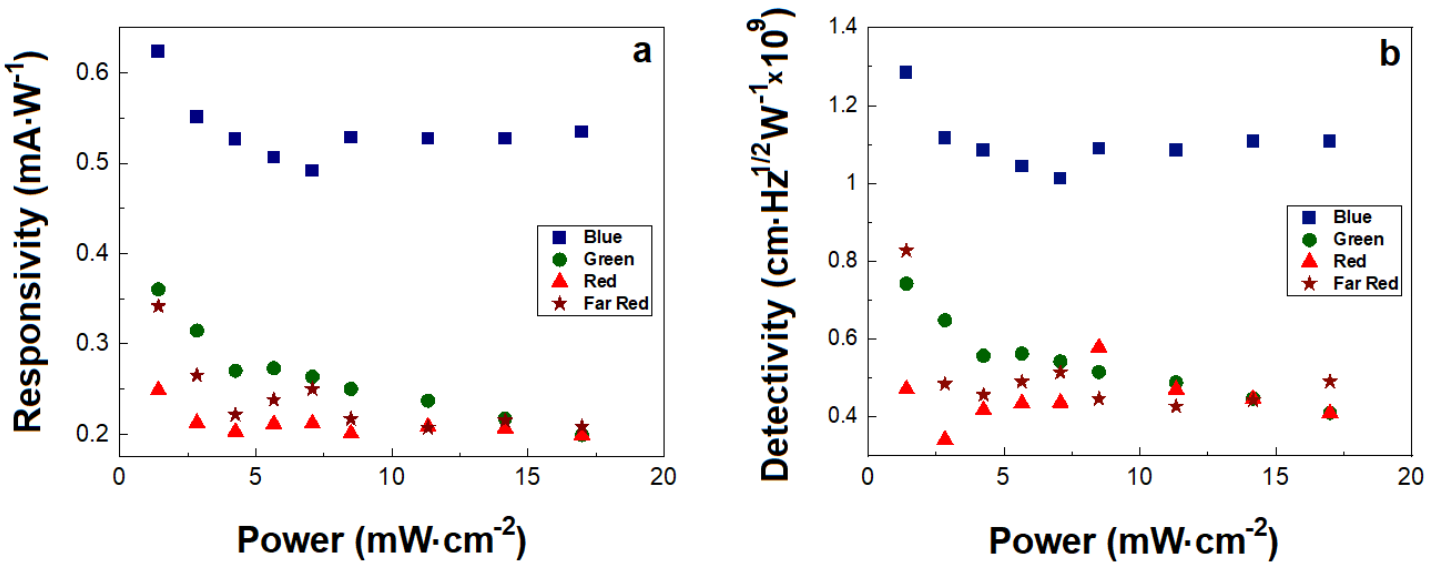

Figure 9. The responsivity (a) and specific detectivity (b) of $\mathrm{TaS}_{2}$-integrated $\mathrm{PD}$ in $0.5 \mathrm{M} \mathrm{H}_{2} \mathrm{SO}_{4}$ solution as a function of power density upon four different illumination wavelengths: blue, green, red and far-red.

Prepared $\mathrm{TaS}_{2}$-based PD exhibited a broadband photodetection capability with the highest photoresponsivity $\left(0.68 \mathrm{~mA} \mathrm{~W}^{-1}\right)$ and specific detectivity $\left(1.39 \times 10^{9} \mathrm{~cm} \mathrm{~Hz}^{1 / 2} \mathrm{~W}^{-1}\right)$ toward $420 \mathrm{~nm}$ light illumination, which is nearly double compared with 532, 630 and $720 \mathrm{~nm}$ illumination wavelengths. The results demonstrate the versatility of $2 \mathrm{H}-\mathrm{TaS}_{2}$-based PEC which exhibits metallike characteristics coupled with broadband light absorption and thus emission due to energy separated bands. The original metallicity of the $\mathrm{TaS}_{2}$ that is assisted by under-coordinated atoms at edge sites is enhanced by the random twisting between the $\mathrm{TaS}_{2}$ monolayers which form a rich homogeneous interface with enhanced conductivity. ${ }^{22}$ This consequently increases the electron transfer in the "TaS 2 electrode $/ 0.5 \mathrm{M} \mathrm{H}_{2} \mathrm{SO}_{4}$ electrolyte" system and thus improves the catalytic performance of the material, establishing it favorable for electrocatalysis. ${ }^{44,1,2}$ 


\section{CONCLUSIONS}

The light-induced efficiency of electrochemically exfoliated $\mathrm{TaS}_{2}$ for photoelectrocatalysis and PEC-based photodetector performance has been demonstrated for the first time. A wellcontrolled exfoliation process that is based on the $\mathrm{TBA}^{+}$-cation intercalation between the $\mathrm{TaS}_{2}$ interlayers was performed in the non-aqueous DMF medium applying a potential of-3.8 V. Fewlayer $\mathrm{TaS}_{2}$ nanosheets of different lateral sizes namely, 10-100 $\mathrm{nm}$ and 1-30 $\mu \mathrm{m}$, were identified and characterized by numerous characterization and microscopy techniques. It was shown that exfoliated $\mathrm{TaS}_{2}$ nanosheets possess a trigonal prismatic structure $(2 \mathrm{H}$ type, hexagonal) and thus, metallic character. The metallicity of the $2 \mathrm{H}-\mathrm{TaS}_{2}$ nanosheets was enhanced by the twisting of the individual flakes and their mutual interaction causing charge density redistribution. This ability was further increased by the external illumination of the light of the following wavelengths: 420 nm (blue), $532 \mathrm{~nm}$ (green), $630 \mathrm{~nm}$ (red) and $720 \mathrm{~nm}$ (far-red). Based on the above, $\mathrm{TaS}_{2}$ nanosheets were tested as PEC for HER and PD of visible light. The as-exfoliated $\mathrm{TaS}_{2}$-based catalyst under light illumination demonstrated high HER catalytic activity with the overpotential for $10 \mathrm{~mA} \cdot \mathrm{cm}^{-2}$ below $575 \mathrm{mV}$ which is $\sim 100 \mathrm{mV}$ lower than during dark mode. Additionally, it was proven that the accurate preparation of the $\mathrm{TaS}_{2}$-based catalyst via sample centrifugation can significantly enhance the catalytic performance of the material. Solution-processed $\mathrm{TaS}_{2}$-enabled PD exhibited broadband light sensitivity in the visible range. The highest photoresponsivity $\left(0.68 \mathrm{~mA} \mathrm{~W}^{-1}\right)$ and specific detectivity $\left(1.39 \times 10^{9} \mathrm{~cm} \mathrm{~Hz}^{1 / 2} \mathrm{~W}^{-1}\right)$ were observed under blue light illumination which was nearly double in comparison to green, red and far-red lights. These findings can potentially ignite multiple insights of $2 \mathrm{H}-\mathrm{TaS}_{2}$ in the photo-induced electrochemistry and (nano)optoelectronics. 


\section{AUTHOR INFORMATION}

\section{Corresponding Author}

Evgeniya Kovalska - Department of Inorganic Chemistry, University of Chemistry and Technology

Prague, Technická 5, 16628 Prague 6, Czech Republic; https://orcid.org/0000-0002-8996-0790.

Email: evgeniya.kovalska.ua@gmail.com

Zdenek Sofer - Department of Inorganic Chemistry, University of Chemistry and Technology Prague, Technická 5, 16628 Prague 6, Czech Republic; https://orcid.org/0000-0002-1391-4448.

Email: soferz@vscht.cz

\section{Authors}

Pradip Kumar Roy - Department of Inorganic Chemistry, University of Chemistry and Technology Prague, Technická 5, 16628 Prague 6, Czech Republic.

Nikolas Antonatos - Department of Inorganic Chemistry, University of Chemistry and Technology Prague, Technická 5, 16628 Prague 6, Czech Republic.

Vlastimil Mazanek - Department of Inorganic Chemistry, University of Chemistry and Technology Prague, Technická 5, 16628 Prague 6, Czech Republic.

Martin Vesely - Department of Organic Technology, University of Chemistry and Technology Prague, Technická 5, 16628 Prague 6, Czech Republic.

Bing Wu - Department of Inorganic Chemistry, University of Chemistry and Technology Prague, Technická 5, 16628 Prague 6, Czech Republic. 


\section{Funding Sources}

Czech Science Foundation (GACR No. 19-26910X).

\section{ACKNOWLEDGMENT}

The authors acknowledge the financial support provided by the Czech Science Foundation (GACR No. 19-26910X).

\section{REFERENCES}

1 Voiry, D. et al. Conducting $\mathrm{MoS}_{2}$ Nanosheets as Catalysts for Hydrogen Evolution Reaction. Nano Letters 13, 6222-6227, doi:10.1021/nl403661s (2013).

2 Voiry, D. et al. Enhanced catalytic activity in strained chemically exfoliated $\mathrm{WS}_{2}$ nanosheets for hydrogen evolution. Nature Materials 12, 850-855, doi:10.1038/nmat3700 (2013).

3 Li, Y., Li, Y.-L., Araujo, C. M., Luo, W. \& Ahuja, R. Single-layer $\mathrm{MoS}_{2}$ as an efficient photocatalyst. Catalysis Science \& Technology 3, 2214-2220, doi:10.1039/C3CY00207A (2013).

4 Bai, S., Wang, L., Chen, X., Du, J. \& Xiong, Y. Chemically exfoliated metallic $\mathrm{MoS}_{2}$ nanosheets: A promising supporting co-catalyst for enhancing the photocatalytic performance of $\mathrm{TiO}_{2}$ nanocrystals. Nano Research 8, 175-183 (2015).

5 Liu, Q. et al. Stable Metallic 1T-WS 2 Nanoribbons Intercalated with Ammonia Ions: The Correlation between Structure and Electrical/Optical Properties. Advanced Materials 27, 4837-4844, doi:10.1002/adma.201502134 (2015).

6 Tsai, C., Chan, K., Nørskov, J. K. \& Abild-Pedersen, F. Theoretical insights into the hydrogen evolution activity of layered transition metal dichalcogenides. Surface Science 640, 133-140, doi:https://doi.org/10.1016/j.susc.2015.01.019 (2015).

7 Johari, P. \& Shenoy, V. B. Tuning the Electronic Properties of Semiconducting Transition Metal Dichalcogenides by Applying Mechanical Strains. ACS Nano 6, 5449-5456, doi:10.1021/nn301320r (2012). 
8 Chhowalla, M. et al. The chemistry of two-dimensional layered transition metal dichalcogenide nanosheets. Nature chemistry 5, 263-275, doi:10.1038/nchem.1589 (2013).

9 Tan, C. \& Zhang, H. Two-dimensional transition metal dichalcogenide nanosheet-based composites. Chemical Society Reviews 44, 2713-2731, doi:10.1039/C4CS00182F (2015).

10 Chiritescu, C. et al. Ultralow thermal conductivity in disordered, layered $\mathrm{WSe}_{2}$ crystals. Science (New York, N.Y.) 315, 351-353, doi:10.1126/science.1136494 (2007).

11 Li, H. et al. Mechanical Exfoliation and Characterization of Single- and Few-Layer Nanosheets of $\mathrm{WSe}_{2}, \mathrm{TaS}_{2}$, and $\mathrm{TaSe}_{2}$. Small 9, 1974-1981, doi:10.1002/smll.201202919 (2013).

12 Peng, J.-P. et al. Molecular beam epitaxy growth and scanning tunneling microscopy study of $\mathrm{TiSe}_{2}$ ultrathin films. Physical Review B 91, 121113, doi:10.1103/PhysRevB.91.121113 (2015).

13 Wang, J. et al. Controlled Synthesis of Two-Dimensional 1T-TiSe2 with Charge Density Wave Transition by Chemical Vapor Transport. Journal of the American Chemical Society 138, 1621616219, doi:10.1021/jacs.6b10414 (2016).

14 Joe, Y. I. et al. Emergence of charge density wave domain walls above the superconducting dome in 1T-TiSe 2. Nature Physics 10, 421-425, doi:10.1038/nphys2935 (2014).

$15 \mathrm{Xi}$, X. et al. Strongly enhanced charge-density-wave order in monolayer $\mathrm{NbSe}_{2}$. Nature Nanotechnology 10, 765-769, doi:10.1038/nnano.2015.143 (2015).

$16 \mathrm{Xi}, \mathrm{X}$. et al. Ising pairing in superconducting $\mathrm{NbSe}_{2}$ atomic layers. Nature Physics 12, 139-143, doi:10.1038/nphys3538 (2016).

17 Sun, S. et al. Direct observation of an optically induced charge density wave transition in $\mathrm{TaSe}_{2}$. Physical Review B 92, 224303, doi:10.1103/PhysRevB.92.224303 (2015).

$18 \mathrm{Yu}$, Y. et al. Gate-tunable phase transitions in thin flakes of $1 \mathrm{~T}-\mathrm{TaS}_{2}$. Nature Nanotechnology 10, 270276, doi:10.1038/nnano.2014.323 (2015).

19 Hovden, R. et al. Atomic lattice disorder in charge-density-wave phases of exfoliated dichalcogenides (1T-TaS $)_{2}$. Proceedings of the National Academy of Sciences 113, 11420-11424, doi:10.1073/pnas.1606044113 (2016). 
20 Sipos, B. et al. From Mott state to superconductivity in $1 \mathrm{~T}-\mathrm{TaS}_{2}$. Nature Materials 7, 960-965, doi:10.1038/nmat2318 (2008).

21 Tsen, A. W. et al. Structure and control of charge density waves in two-dimensional 1T-TaS 2 . Proceedings of the National Academy of Sciences 112, 15054, doi:10.1073/pnas.1512092112 (2015).

22 Pan, J. et al. Enhanced Superconductivity in Restacked $\mathrm{TaS}_{2}$ Nanosheets. Journal of the American Chemical Society 139, 4623-4626, doi:10.1021/jacs.7b00216 (2017).

23 Navarro-Moratalla, E. et al. Enhanced superconductivity in atomically thin $\mathrm{TaS}_{2}$. Nature Communications 7, 11043, doi:10.1038/ncomms11043 (2016).

24 Peng, J. et al. Disorder Enhanced Superconductivity toward $\mathrm{TaS}_{2}$ Monolayer. ACS Nano 12, 94619466, doi:10.1021/acsnano.8b04718 (2018).

25 Zong, P.-A. et al. Flexible Foil of Hybrid $\mathrm{TaS}_{2} /$ Organic Superlattice: Fabrication and Electrical Properties. Small 16, 1901901, doi:10.1002/smll.201901901 (2020).

$26 \mathrm{Wu}, \mathrm{X}$. -C., Tao, Y.-R. \& Gao, Q.-X. Fabrication of $\mathrm{TaS}_{2}$ nanobelt arrays and their enhanced fieldemission. Chemical Communications, 6008-6010, doi:10.1039/B913935D (2009).

27 Yoshida, M. et al. Controlling charge-density-wave states in nano-thick crystals of 1T-TaS 2 . Scientific Reports 4, 7302, doi:10.1038/srep07302 (2014).

28 Tsen, A. W. et al. Structure and control of charge density waves in two-dimensional $1 \mathrm{~T}-\mathrm{TaS}_{2}$. Proceedings of the National Academy of Sciences 112, 15054-15059, doi:10.1073/pnas.1512092112 (2015).

$29 \mathrm{Hu}$, Y. et al. Toward Exploring the Structure of Monolayer to Few-layer $\mathrm{TaS}_{2}$ by Efficient Ultrasoundfree Exfoliation. Nanoscale Research Letters 13, 20, doi:10.1186/s11671-018-2439-z (2018).

30 Nguyen, T. P. et al. Transition Metal Disulfide Nanosheets Synthesized by Facile Sonication Method for the Hydrogen Evolution Reaction. The Journal of Physical Chemistry C 120, 3929-3935, doi:10.1021/acs.jpcc.5b12164 (2016).

31 Sutto, T. E. \& Averill, B. A. in Lower-Dimensional Systems and Molecular Electronics (eds Robert M. Metzger, Peter Day, \& George C. Papavassiliou) 289-291 (Springer US, 1990). 
32 Zhou, L. et al. Tantalum disulfide quantum dots: preparation, structure, and properties. Nanoscale Research Letters 15, 20, doi:10.1186/s11671-020-3250-1 (2020).

33 Kovalska, E. et al. Non-aqueous solution-processed phosphorene by controlled low-potential electrochemical exfoliation and thin film preparation. Nanoscale 12, 2638-2647, doi:10.1039/c9nr10257d (2020).

34 Kovalska, E., Antonatos, N., Luxa, J. \& Sofer, Z. “Top-down” Arsenene Production by Low-Potential Electrochemical Exfoliation. Inorganic Chemistry 59, 11259-11265, doi:10.1021/acs.inorgchem.0c00243 (2020).

35 Kovalska, E. et al. Single-Step Synthesis of Platinoid-Decorated Phosphorene: Perspectives for Catalysis, Gas Sensing, and Energy Storage. ACS Applied Materials \& Interfaces, doi:10.1021/acsami.0c15525 (2020).

36 Yin, H.-J., Zhou, J.-H. \& Zhang, Y.-W. Shaping well-defined noble-metal-based nanostructures for fabricating high-performance electrocatalysts: advances and perspectives. Inorganic Chemistry Frontiers 6, 2582-2618, doi:10.1039/C9QI00689C (2019).

37 Konanki, S. S. \& Beyette, F. R. in 2000 Digest of the LEOS Summer Topical Meetings. ElectronicEnhanced Optics. Optical Sensing in Semiconductor Manufacturing. Electro-Optics in Space. Broadband Optical Networks (Cat. No.00TH8497). I13-I14.

38 Sung, S. H., Schnitzer, N., Brown, L., Park, J. \& Hovden, R. Stacking, strain, and twist in 2D materials quantified by 3D electron diffraction. Physical Review Materials 3, 064003, doi:10.1103/PhysRevMaterials.3.064003 (2019).

39 Li, Y., Xiao, H., Zhou, P. \& Cao, J. Electronic structures of twist-stacked 1T-TaS 2 bilayers. Physics Letters A 383, 2302-2308, doi:https://doi.org/10.1016/j.physleta.2019.04.043 (2019).

40 Wang, X. H. et al. Antimonene: A Promising Candidate for $\mathrm{SF}_{6}$ Decomposition Gas Sensors With High Sensitivity and High Stability. IEEE Electron Device Letters 41, 1408-1411, doi:10.1109/LED.2020.3012693 (2020). 
41 Wang, K. et al. Electrical control of charged carriers and excitons in atomically thin materials. Nature Nanotechnology 13, 128-132, doi:10.1038/s41565-017-0030-х (2018).

42 Hirata, T. \& Ohuchi, F. S. Temperature dependence of the Raman spectra of $1 \mathrm{~T}-\mathrm{TaS}_{2}$. Solid State Communications 117, 361, doi:10.1016/s0038-1098(00)00468-3 (2001).

$43 \mathrm{Wu}$, D. et al. Ultrabroadband photosensitivity from visible to terahertz at room temperature. J Science Advances 4, eaao3057, doi:10.1126/sciadv.aao3057 \% (2018).

44 Luxa, J. et al. $2 \mathrm{H} \rightarrow 1$ T Phase Engineering of Layered Tantalum Disulfides in Electrocatalysis: Oxygen Reduction Reaction. 23, 8082-8091, doi:10.1002/chem.201701494 (2017).

45 Yuan, Y.-P., Ruan, L.-W., Barber, J., Joachim Loo, S. C. \& Xue, C. Hetero-nanostructured suspended photocatalysts for solar-to-fuel conversion. Energy \& Environmental Science 7, 3934-3951, doi:10.1039/C4EE02914C (2014).

46 Paul, K. K. et al. Strongly enhanced visible light photoelectrocatalytic hydrogen evolution reaction in an n-doped $\mathrm{MoS}_{2} / \mathrm{TiO}_{2}$ (B) heterojunction by selective decoration of platinum nanoparticles at the $\mathrm{MoS}_{2}$ edge sites. Journal of Materials Chemistry A 6, 22681-22696, doi:10.1039/C8TA06783J (2018).

$47 \mathrm{Lu}$, Y. et al. Hierarchical $\mathrm{CdS} / \mathrm{m}-\mathrm{TiO}_{2} / \mathrm{G}$ ternary photocatalyst for highly active visible light-induced hydrogen production from water splitting with high stability. Nano Energy 47, 8-17, doi:https://doi.org/10.1016/j.nanoen.2018.02.021 (2018).

48 Najafi, L. et al. $\mathrm{TaS}_{2}, \mathrm{TaSe}_{2}$, and Their Heterogeneous Films as Catalysts for the Hydrogen Evolution Reaction. ACS Catalysis 10, 3313-3325, doi:10.1021/acscatal.9b03184 (2020).

$49 \mathrm{Li}, \mathrm{H}$. et al. Atomic-Sized Pores Enhanced Electrocatalysis of $\mathrm{TaS}_{2}$ Nanosheets for Hydrogen Evolution. 28, 8945-8949, doi:10.1002/adma.201602502 (2016).

50 Shi, J. et al. Two-dimensional metallic tantalum disulfide as a hydrogen evolution catalyst. Nature Communications 8, 958, doi:10.1038/s41467-017-01089-z (2017).

51 Tong, T. et al. Sensitive and Ultrabroadband Phototransistor Based on Two-Dimensional $\mathrm{Bi}_{2} \mathrm{O}_{2} \mathrm{Se}$ Nanosheets. 29, 1905806, doi:10.1002/adfm.201905806 (2019). 
52 Wen, J. et al. Ultra-broadband self-powered reduced graphene oxide photodetectors with annealing temperature-dependent responsivity.

Carbon

153, 274-284, doi:https://doi.org/10.1016/j.carbon.2019.07.033 (2019).

53 Long, M. et al. Room temperature high-detectivity mid-infrared photodetectors based on black arsenic phosphorus. 3, e1700589, doi:10.1126/sciadv.1700589 \%J Science Advances (2017).

54 Bera, K. P. et al. Graphene Sandwich Stable Perovskite Quantum-Dot Light-Emissive Ultrasensitive and Ultrafast Broadband Vertical Phototransistors. ACS Nano 13, 12540-12552, doi:10.1021/acsnano.9b03165 (2019). 